\title{
A retrospective study of emergency department potassium disturbances: severity, treatment, and outcomes
}

\author{
Adam J Singer ${ }^{1}$, Henry C Thode Jr ${ }^{1}$, W Frank Peacock ${ }^{2}$ \\ 'Department of Emergency Medicine, Stony Brook University, Stony Brook, NY, USA \\ ${ }^{2}$ Department of Emergency Medicine, Baylor College of Medicine, Houston, TX, USA
}

Objective Disturbances in potassium $(K)$ levels are relatively common and may be associated with significant morbidity and mortality; however, treatments vary. Our purpose was to determine the incidence, treatments, and outcomes associated with hyperkalemia and hypokalemia in emergency department (ED) patients.

Methods We performed a structured, retrospective review of electronic medical records of consecutive adult ED patients with $\mathrm{K}$ measured while in the ED. Demographic, clinical, and laboratory data as well as treatments, disposition, and in-hospital complications were collected. Univariate and multivariate analyses, presented as adjusted odds ratios, were used to compare outcomes by $\mathrm{K}$ levels.

Results Of 100,260 visits in 2014, an ED K level was ordered in 48,827 (49\%). A total of 1,738 patients (3.6\%) were excluded because of sample hemolysis. The $\mathrm{K}$ was low $(<3.5 \mathrm{mEq} / \mathrm{L})$ in $5.5 \%$, normal (3.5 to $5.0 \mathrm{mEq} / \mathrm{L}$ ) in $90.9 \%$, and elevated $(>5.0 \mathrm{mEq} / \mathrm{L}$ ) in $3.6 \%$ of patients. Patients with hyperkalemia were older (64 vs. 49 years, $\mathrm{P}<0.001)$ and more likely male $(58 \%$ vs. $40 \%, P<0.001)$. Treatment for hyperkalemia varied greatly. After adjusting for confounders, both hyperkalemia and hypokalemia were associated with inpatient hospitalization and death. At least one medication was used to manage hyperkalemia in $11.5 \%$ of patients with a $\mathrm{K}$ of 5.1 to $5.4 \mathrm{mEq} / \mathrm{L}, 36.4 \%$ of those with a $\mathrm{K} 5.5$ to $6 \mathrm{mEq} / \mathrm{L}$ and $77.0 \%$ of the cohort with $\mathrm{K}>6 \mathrm{mEq} / \mathrm{L}$.

Conclusion Hyperkalemia or hypokalemia occur in 1 of 11 ED patients and are associated with inpatient admission and mortality. Treatment of hyperkalemia varies greatly suggesting the need for evidence-based treatment guidelines.

Keywords Hyperkalemia; Hypokalemia; Mortality; Admission; Emergency service, hospital

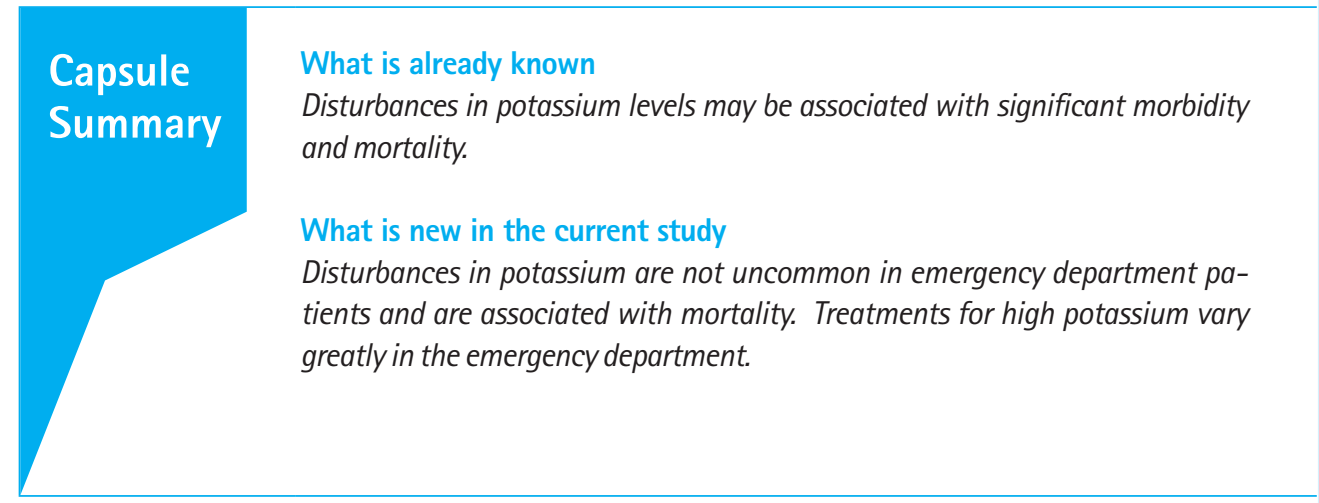

elSSN: 2383-4625

Received: 19 November 2016 Revised: 21 December 2016 Accepted: 22 December 2016

Correspondence to: Adam J Singer Department of Emergency Medicine, Stony Brook University, HSC-L4-080, Stony Brook, NY 11794, USA

E-mail: adam.singer@stonybrook.edu

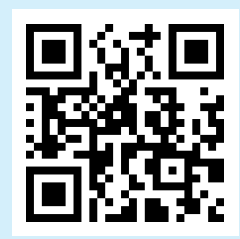

How to cite this article:

Singer AJ, Thode HC Jr, Peacock WF. A retrospective study of emergency department potassium disturbances: severity, treatment, and outcomes. Clin Exp Emerg Med 2017;4(2):73-79.

This is an Open Access article distributed under the terms of the Creative Commons Attribution Non-Commercial License (http:// creativecommons.org/licenses/by-nc/4.0/). 


\section{INTRODUCTION}

Hyperkalemia is one of the most common and potentially lethal electrolyte abnormalities. Prior studies estimated that it occurs in approximately $1 \%$ to $10 \%$ of hospitalized patients. ${ }^{1-3}$ Depending on its definition, studies limited to the emergency department (ED) have reported rates of hyperkalemia from $0.36 \%$ to $2.6 \% .^{4-6}$ Regardless of the exact definition, multiple studies have demonstrated an association of hyperkalemia with morbidity and mortality, mostly due to lethal cardiac dysrhythmias. ${ }^{7-11}$

Studies regarding the incidence and outcomes associated with hypokalemia in the ED are limited. Marti et al. ${ }^{12}$ reported that of 43,805 ED patients with serum potassium measurement, 4,826 $(11 \%)$ had a potassium less than $3.5 \mathrm{mEq} / \mathrm{L}$ at presentation. Fiftythree patients had severe hypokalemia $(<2.6 \mathrm{mEq} / \mathrm{L})$, of which 26 (49\%) were symptomatic and 20 (69\%) had electrocardiogram changes. While 9 patients died in the hospital, there was no difference in potassium levels between those who died and survivors. ${ }^{12}$ An inpatient based study by Goyal et al., ${ }^{10}$ describing outcomes associated with serum potassium levels in 38,689 patients with an acute myocardial infarction, found that the mortality rate increased to $46.2 \%$ when the serum potassium decreased below $3.0 \mathrm{mEq} / \mathrm{L}$.

The recent development of several novel oral therapies for elevated potassium has led to renewed interest in hyperkalemia. ${ }^{13,14}$ Additionally, an increase in the number of patients on potassium increasing drugs, such as angiotensin-converting enzyme inhibitors, angiotensin receptor blockers and spironolactone has led to an increase in the number of patients who present to the ED with hyperkalemia. ${ }^{15-17}$ Conversely, the current heart failure epidemic potentially leads to increasing rates of ED hypokalemia presentations due to excessive loop diuretic therapy. Ultimately, emergency physicians can expect to encounter more and more patients with potassium disturbances in the years to come.

The goals of the current study were to determine the incidence, common treatments and outcomes of potassium disturbances in ED patients.

\section{METHODS}

\section{Study design}

We conducted a structured, ${ }_{1}^{18,19}$ retrospective medical record review of all patients presenting to the ED for whom a potassium level was documented. The study was approved by the institutional review board and exempt from written informed consent due to it retrospective nature and minimal risk.

\section{Setting}

The study was conducted at a tertiary care, academic medical center with an affiliated residency in emergency medicine and an annual ED census of approximately 100,000. The medical center is also a major referral center for a county of 1.5 million inhabitants. Our hospital has no formal protocol or policy for managing patients with potassium disturbances.

\section{Patients}

All patients were included if they presented to the ED during the 2014 calendar year, including those with trauma and cardiac arrest. Because of the challenges in treating hyperkalemia, while general data were obtained for all patients, additional data were obtained in patients with hyperkalemia, defined as a potassium level of greater than $5.0 \mathrm{mEq} / \mathrm{L}$. Patients whose blood specimen was hemolyzed, were excluded.

\section{Study protocol}

A computerized search of the electronic medical records was performed of all ED visits during 2014 in which a potassium level was reported in the laboratory data base. Potassium levels were categorized as low ( $<3.5 \mathrm{mEq} / \mathrm{L})$, normal ( 3.5 to $5.0 \mathrm{mq} / \mathrm{L}$ ) or high ( $>5.0 \mathrm{mEq} / \mathrm{L}$ ) based on the definitions of the American Heart Association. ${ }^{20}$ Potassium level was defined based on the first reported potassium result.

Data collection included baseline demographic, admissions, clinical information, medications used, and treatments received while in the ED. All study variables were defined a priori and documented in a coding guide for the chart abstractors. A standard data collection instrument was used. All data abstractors received specific training in the definitions and use of the data collection instrument. Monitoring of data abstraction was performed periodically by the principal investigator, and a subset of 20 randomly selected charts were independently reviewed by two abstractors to determine inter-observer agreement on the primary outcome.

\section{Outcomes}

The primary outcome was mortality during the index hospitalization, both in the ED and during in-patient hospitalization. Other outcomes were rates of admission to an in-patient floor or an intensive care unit, ED length of stay (LOS), and overall hospital length LOS in those patients admitted to the hospital.

\section{Data analysis}

For descriptive statistics, we reported continuous variables with their mean (standard deviation) values and binary data as numbers (percentages). Univariate comparisons across potassium groups 
were performed using chi-square and Fisher exact tests for binary data, and analysis of variance or Mann-Whitney U-tests for continuous data, as appropriate. Adjusted admission and mortality rates were calculated using logistic regression adjusting for age, gender, diabetes, heart failure, chronic kidney failure. septicemia, sodium, and creatinine.

\section{RESULTS}

In 2014 there were 100,260 patient visits to the ED. Of these, 48,827 (49\%) had at least one serum potassium lab test ordered, excluding urine tests. Mean (standard deviation) age of these patients was 49 (22), and 46\% were male.

Of the 48,827 patients with a potassium lab test ordered, 1,738 $(3.6 \%)$ did not get a test value back, mostly due to hemolysis of the samples, and were excluded from further analysis. The mean age (standard deviation) of the remaining 47,089 patients was 49 (22) years, and 46\% were male (Table 1). Common diagnostic and comorbidity categories included hypertension (30\%), lipid disorders (22\%), trauma (17\%), and non-dependent drug abuse (17\%). Other comorbidities included diabetes (16\%), heart failure (8\%), chronic renal failure (7\%), and septicemia (4\%).

Median (interquartile range) ED LOS was 7.1 (4.8 to 10.9) hours. Of all patients included in the analysis, 40\% were admitted, $2.7 \%$ to an intensive care unit, and $1 \%$ died. Most potassium levels were normal, 42,818 (91\%), with only 4,271 (9.1\%) having abnormal results. Of the abnormal potassium results, 2,574 $(5.5 \%)$ had a low value $(<3.5 \mathrm{mEq} / \mathrm{L})$, and 1,697 (3.6\%) had a re-

Table 1. Characteristics of study population $(n=47,089)$

\begin{tabular}{lc}
\hline Characteristics & Value \\
\hline Mean age (SD) & $49(22)$ \\
Male & $21,573(46)$ \\
Discharge diagnosis hyperkalemia & $772(1.6)$ \\
ED disposition & \\
Median ED LOS (IOR, hr) & $7.1(4.8-10.9)$ \\
Admitted & $19,014(40.4)$ \\
Admitted to ICU & $1,252(2.7)$ \\
Died at index visit & $489(1.0)$ \\
Abnormal potassium level (mEq/L) & $4,271(9.1)$ \\
Low (<3.5) & $2,574(5.5)$ \\
Normal (3.5-5.0) & $42,818(90.9)$ \\
Minimally elevated (5.1-5.4) & $1,058(2.2)$ \\
Moderately elevated (5.5-6.0) & $448(1.0)$ \\
Severely elevated (>6.0) & $191(0.4)$ \\
\hline
\end{tabular}

Values are presented as number (\%) unless otherwise indicated. $S D$, standard deviation; ED, emergency department; LOS, length of stay; IQR, interquartile range; ICU, intensive care unit.

Table 2. Patient characteristics, outcomes, and treatments by potassium level $(\mathrm{mEq} / \mathrm{L})$

\begin{tabular}{|c|c|c|c|c|c|c|}
\hline & $\begin{array}{l}\text { Low } \\
(<3.5)\end{array}$ & $\begin{array}{c}\text { Normal } \\
(3.5-5.0)\end{array}$ & $\begin{array}{c}\text { Minimal elevation } \\
(5.1-5.4)\end{array}$ & $\begin{array}{c}\text { Moderate elevation } \\
(5.5-6.0)\end{array}$ & $\begin{array}{c}\text { Severe elevation } \\
(>6.0)\end{array}$ & P-value \\
\hline$\%$ male & 34 & 46 & 58 & 57 & 56 & $<0.001$ \\
\hline Mean age (SD) & $48(21)$ & $49(22)$ & $62(24)$ & $61(26)$ & $61(23)$ & $<0.001$ \\
\hline Mean MEWS (SD) & $1.7(1.7)$ & $1.4(1.5)$ & $2.1(1.8)$ & $2.5(2.1)$ & $3.1(2.6)$ & $<0.001$ \\
\hline Diabetes & 11.7 & 15.3 & 40.6 & 44.9 & 41.4 & $<0.001$ \\
\hline Chronic kidney disease & 4.7 & 6.2 & 35.1 & 46.9 & 56.5 & $<0.001$ \\
\hline Heart failure & 5.7 & 7.3 & 27.5 & 29.0 & 30.9 & $<0.001$ \\
\hline Septicemia & 5.8 & 3.3 & 11.2 & 11.2 & 18.8 & $<0.001$ \\
\hline$\%$ creatinine $>1.5 \mathrm{mg} / \mathrm{dL}$ & 6.1 & 5.9 & 39.8 & 57.2 & 73.5 & $<0.001$ \\
\hline$\%$ glucose $>150 \mathrm{mg} / \mathrm{dL}$ & 14.1 & 13.0 & 29.4 & 37.5 & 47.1 & $<0.001$ \\
\hline \% sodium < $135 \mathrm{mEq} / \mathrm{L}$ & 11.3 & 6.3 & 17.6 & 26.0 & 28.8 & $<0.001$ \\
\hline \% sodium $>145 \mathrm{mEq} / \mathrm{L}$ & 2.3 & 1.4 & 1.8 & 3.1 & 4.7 & \\
\hline \multicolumn{7}{|l|}{ Outcomes } \\
\hline$\%$ admitted & 48 & 39 & 69 & 80 & 86 & $<0.001$ \\
\hline$\%$ died & 1.9 & 0.8 & 3.9 & 5.5 & 13.6 & $<0.001$ \\
\hline \multicolumn{7}{|l|}{ Treatments ( $\%$ of total patients) } \\
\hline Any $\mathrm{K}^{+}$reducing medication & 3.0 & 4.2 & 11.5 & 36.4 & 77.0 & \\
\hline Salbutamol & 1.6 & 2.5 & 3.5 & 7.1 & 17.8 & \\
\hline $\mathrm{NaHCO}_{3}$ & 0.3 & 0.2 & 1.5 & 8.5 & 29.0 & \\
\hline Insulin & 0.9 & 1.4 & 5.4 & 19.0 & 64.4 & \\
\hline Calcium & 0.3 & 0.1 & 0.5 & 4.0 & 24.6 & \\
\hline Dialysis & 0.4 & 0.4 & 3.5 & 6.3 & 25.1 & \\
\hline Potassium & 30.7 & 2.8 & 0 & 0 & 0 & \\
\hline
\end{tabular}

SD, standard deviation; MEWS, modified early warning scores. 
sult in excess of $5.0 \mathrm{mEq} / \mathrm{L}$. Subcategorizing high potassium levels into minimally elevated ( 5.1 to $5.4 \mathrm{mEq} / \mathrm{L}$ ), moderately elevated ( 5.5 to $6.0 \mathrm{mEq} / \mathrm{L}$ ), and severely elevated (> $6.0 \mathrm{mEq} / \mathrm{L}$ ) resulted in 1,058 (2.2\%), 448 (1.0\%), and 191 (0.4\%) in each cohort, respectively. Co-morbidities, such as diabetes mellitus, chronic kidney disease, septicemia, and heart failure were common in patients with elevated potassium, and increased rates occurred with higher potassium levels (Table 2). Modified early warning scores (MEWS) showed a similar pattern, with a slight increase for patients with hypokalemia compared to normal, and increasing MEWS scores with increasing levels of potassium above normal.

Both age and gender were related to potassium level (Table 2). Age was positively associated with potassium level $(P<0.001)$, while males were more likely to have elevated potassium and females more likely to have low potassium $(\mathrm{P}<0.001)$. Potassium levels were also associated with admission and mortality (both $\mathrm{P}<0.001$ ) (Table 2). Admission and mortality increased as potassium levels increased relative to normal levels (Figs. 1, 2, respectively). Admission and mortality rates were also higher for patients with low potassium levels compared to those with normal levels but were not as high as those with elevated potassium. Both admission rates and mortality increased as potassium levels became

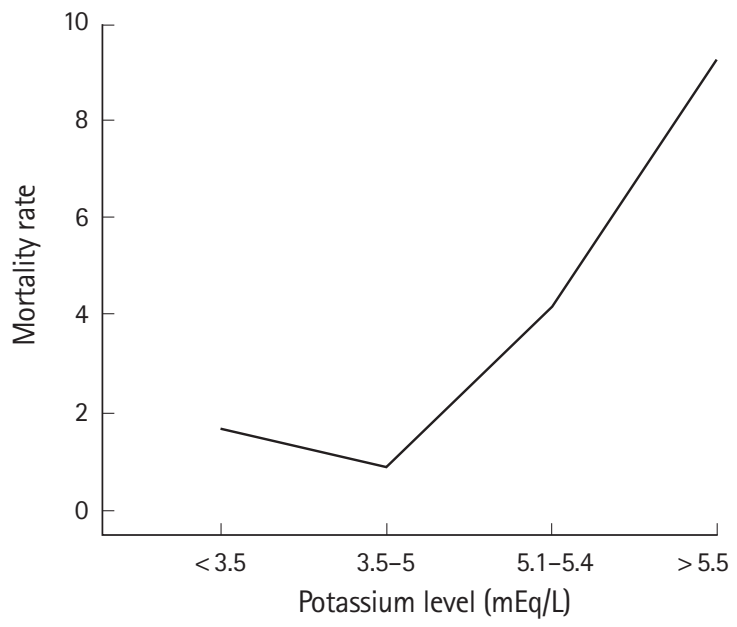

Fig. 1. Association between potassium levels and mortality. increasingly high or low relative to normal. Creatinine, sodium, and glucose were associated with potassium levels.

Medications for treating elevated potassium were given to $4.9 \%$ of patients in the ED. These included Kayexalate, sodium bicarbonate, calcium gluconate, albuterol, insulin, calcium carbonate, calcium chloride, and glucose. Dialysis was performed on $0.6 \%$ of hyperkalemic ED patients. Potassium was given to $4.2 \%$ of patients. All treatments were associated with potassium level (Table 2).

Multivariate analysis of admission and death showed the same associations for increased risk as in the univariate comparisons (Table 3). ED LOS and hospital LOS for admitted patients were longest for patients with elevated potassium levels (Table 3 ).

There were 94 patients with elevated potassium and 50 with hypokalemia who died while in hospital; 4 and 11 died in the ED, respectively. Median (interquartile range) times to death from ED presentation were 103 (21 to 288) hours and 156 (39 to 340), respectively, which were not significantly different. Of all deaths, 10 $(20 \%)$ and $25(27 \%)$ occurred within 24 hours of ED arrival in the two groups, respectively, and another 4 (8\%) and 9 (10\%) within the next 24 hours. Fig. 3 demonstrates the survival curves for those who died. The admitting diagnoses of patients who died are

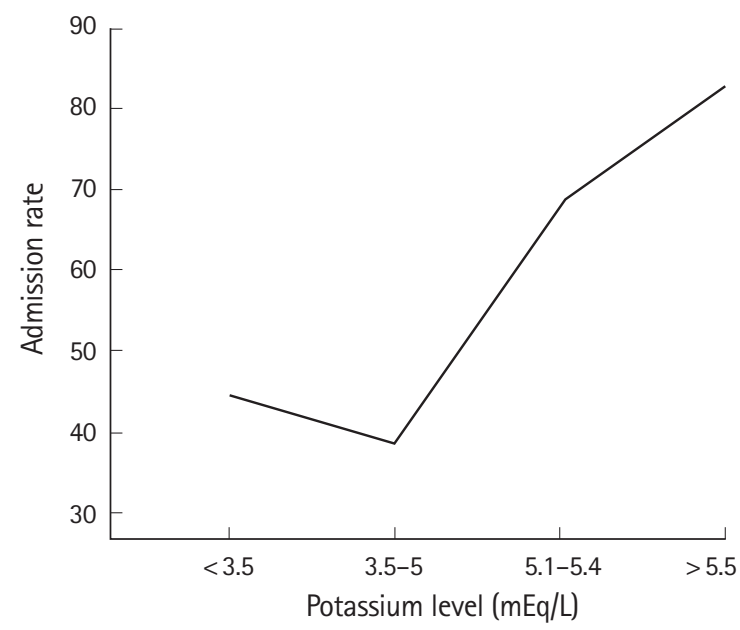

Fig. 2. Association between potassium levels and admission rate.

Table 3. Adjusted outcomes stratified by potassium levels ( $\mathrm{mEq} / \mathrm{L})$

\begin{tabular}{|c|c|c|c|c|c|}
\hline Outcomes & $\begin{array}{l}\text { Low } \\
(<3.5)\end{array}$ & $\begin{array}{l}\text { Normal } \\
(3.5-5.0)\end{array}$ & $\begin{array}{l}\text { Minimally elevated } \\
(5.1-5.4)\end{array}$ & $\begin{array}{l}\text { Moderately elevated } \\
\qquad(5.5-6.0)\end{array}$ & $\begin{array}{l}\text { Severely elevated } \\
\qquad(>6.0)\end{array}$ \\
\hline Inpatient admissions: $\mathrm{aOR}^{\mathrm{a})}(95 \% \mathrm{Cl})$ & $1.6(1.4-1.7)$ & 1.0 (reference) & $1.4(1.2-1.7)$ & $2.6(2.0-3.3)$ & $3.1(2.0-5.0)$ \\
\hline Mortality: aOR (95\% Cl) & $2.4(1.7-3.3)$ & 1.0 (reference) & $1.6(1.1-2.4)$ & $2.9(1.8-4.5)$ & $5.0(2.9-8.6)$ \\
\hline Median (IOR) ED LOS, hours & $7.7(5.2-11.7)$ & $7.0(4.8-10.7)$ & $8.1(5.4-11.8)$ & $8.6(5.9-13.0)$ & $9.5(6.7-13.3)$ \\
\hline $\begin{array}{l}\text { Median (IQR) hospital LOS (hr, admits } \\
\text { discharged alive only) }\end{array}$ & $91(44-170)$ & $70(38-143)$ & $92(48-186)$ & $94(46-209)$ & $124(59-241)$ \\
\hline
\end{tabular}

$a O R$, adjusted odds ratio; $\mathrm{Cl}$, confidence interval; IQR, interquartile range; $E D$, emergency department; $L O S$, length of stay.

${ }^{a)}$ Adjusted for age, gender, chronic kidney failure, heart failure, diabetes, septicemia, creatinine, sodium. 


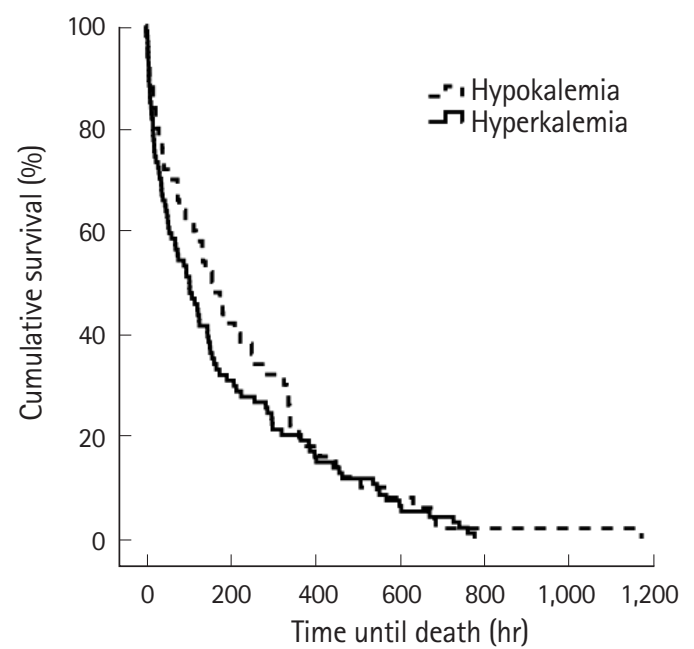

Fig. 3. Survival curve for all deaths in patients with abnormal potassium.

presented in Table 4.

\section{DISCUSSION}

Our study demonstrates that roughly 1 in 11 ED patients present with either high (3.6\%) or low (5.5\%) serum potassium levels. We have also shown that both hyperkalemia and hypokalemia are associated with increased odds of death during the index hospitalization. We further show that the odds of death increases as the potassium further deviates from the normal value resulting in a "U-shaped" mortality curve. Finally, despite its relative high frequency, there is great variability in the management of hyperkalemia in the ED despite similarities in clinical factors within potassium groups such as lab values, MEWS, and comorbidities. While there are multiple textbooks with recommendations for treating hyperkalemia, recommended treatments and thresholds for treatment vary considerably and there is no uniformity. Thus, evidence based guidelines for the management of this potentially lethal condition are greatly needed.

A number of prior studies have evaluated the incidence and etiology of hyperkalemia in the ED. A European study of 38,455 patients presenting to an ED, found that 139 (0.36\%) patients had a potassium level of $>5.2 \mathrm{mEq} / \mathrm{L}$. Major etiologies included renal failure (83\%) and potassium increasing drugs (75\%). ${ }^{4}$ Whether the lower rate of hyperkalemia in the European study is the result of a health care system difference, or some other cause (e.g., different medication prescribing practices), is unclear.

In another study the authors performed a retrospective chart review of ED patients with the laboratory diagnosis of hyperkalemia (potassium level $>6.0 \mathrm{mEq} / \mathrm{L}$ ). ${ }^{21}$ Of $175 \mathrm{ED}$ visits that occurred over a 1-year time period, 168 (96\%) received specific
Table 4. Admitting diagnoses in patient that died in the emergency department or inpatient unit

\begin{tabular}{lccc}
\hline $\begin{array}{l}\text { Diagnostic group/ } \\
\text { comorbidity }\end{array}$ & $\begin{array}{c}\text { Elevated potassium } \\
(\mathrm{n}=94)\end{array}$ & $\begin{array}{c}\text { Low potassium } \\
(\mathrm{n}=50)\end{array}$ & P-value \\
\hline Hypertension & 41 & 34 & 0.38 \\
Septicemia & 48 & 48 & 0.99 \\
Chronic kidney disease & 33 & 8 & 0.001 \\
Heart failure & 40 & 22 & 0.03 \\
Diabetes & 47 & 18 & 0.001 \\
Hypothyroidism & 13 & 4 & 0.09 \\
Drug abuse & 5 & 18 & 0.01 \\
Trauma & 57 & 64 & 0.45 \\
\hline
\end{tabular}

Values are presented as $\%$.

treatment for hyperkalemia. The median (interquartile range) time from triage to initiation of treatment was 117 (59 to 196) minutes. The initial ED treatment was insulin and glucose in 54 (33\%) patient visits; an intravenous calcium infusion (calcium gluconate, 37; calcium chloride, 5) was administered initially in $42(25 \%)$ patients. In this study, two patients died in the ED, however, in-hospital mortality was not reported. ${ }^{21}$ A study conducted by Acker et al., ${ }^{22}$ nearly two decades ago, identified 127 episodes of hyperkalemia during an observation-only phase of a 3.5 month study and another 115 episodes during a phase in which physicians were specifically notified and treatment recommendations were made. In this study treatments included exchange resins (51\%), insulin (46\%) intravenous calcium (36\%), bicarbonate (34\%), and albuterol (4\%). There were no reported deaths and treatments were considered equally efficacious.

We showed that both very low and very high levels of potassium are associated with increased mortality. These findings and a "U" shaped association curve between potassium levels and mortality have been previously reported. Goyal et al. ${ }^{10}$ determined the association between serum potassium levels and in-hospital mortality in 38,689 patients with acute myocardial infarction admitted to one of 67 hospitals between 2000 and 2008. Compared to a reference group with a potassium level between 3.5 to $4.0 \mathrm{mEq} /$ $\mathrm{L}$, the adjusted mortality rates were highest for potassium $>5.5$ $\mathrm{mEq} / \mathrm{L}$ (odds ratio, 2.65; 95\% confidence interval, 1.70 to 4.13 ) and potassium $<3.0 \mathrm{mEq} / \mathrm{L}$ (odds ratio, 2.31; 95\% confidence interval, 0.74 to 7.24$)$. Hayes et al. ${ }^{23}$ studied pre-dialysis mortality and its association with potassium levels in 1,227 males with chronic kidney disease. Of interest, mortality was significantly increased with potassium $<3.8 \mathrm{mEq} / \mathrm{L}$ in both white and black patients, and with potassium $>5.5 \mathrm{mEq} / \mathrm{L}$ in white but not black patients suggesting that black patients may be more tolerant of high potassium than whites. A retrospective study in the ED found that nine of 53 patients (17\%) with severe hypokalemia 
( $<2.6 \mathrm{mEq} / \mathrm{L})$ died after admission. ${ }^{12}$ However, the mortality among patients with higher levels of potassium were not reported. Thus there was no comparison group.

A large retrospective study of 66,259 hyperkalemic events found that 5,945 patients (2.4\%) died within one day of blood draw. $^{24}$ The odds ratio of death was significantly associated with potassium levels for patients both with and without chronic kidney disease. For patients without chronic kidney disease, the odds of death was at least 10 and 31 times greater for patients with moderate (potassium $>5.5$ and $<6.0 \mathrm{mEq} / \mathrm{L}$ ) and severe (potassium > $6.0 \mathrm{mEq} / \mathrm{L}$ ) hyperkalemia, respectively. Interestingly, there tended to be an inverse relationship between the severity (stage) of chronic kidney disease and odds of death, possibly due to chronic adaptation to elevated potassium levels.

The major limitation of the current study is its retrospective nature, which may have led to a selection bias. An ongoing prospective study of patients presenting to the ED with hyperkalemia will address some of these limitations. A prior study has also addressed the accuracy of coding for hyperkalemia. ${ }^{5}$ While having very high specificity (99\%), very poor sensitivity (14.6\%) would have led to a gross underestimation of the incidence of hyperkalemia.

Another limitation is that the analysis is limited to the first measured potassium level after ED presentation. Thus there may have been patients who developed abnormalities in their potassium levels on repeat or subsequent measurements. Further, the current study was conducted at a single, suburban, mostly affluent medical center which may have affected treatment decisions. As such, it is unclear whether these results would generalize to other dissimilar settings.

In conclusion, we have demonstrated that both hyperkalemia and hypokalemia in the ED are associated with an increased risk of death that is proportional to the their severity. We have also shown considerable variability in the management of hyperkalemia. Guidelines for management of disturbances of potassium in the ED are recommended to reduce variability in management.

\section{CONFLICT OF INTEREST}

Adam J Singer and W Frank Peacock have received consulting fees and research support from ZS Pharma.

\section{REFERENCES}

1. Paice B, Gray JM, McBride D, Donnelly T, Lawson DH. Hyperkalaemia in patients in hospital. Br Med J 1983;286:1189-92.

2. Moore ML, Bailey RR. Hyperkalaemia in patients in hospital. $\mathrm{N}$
Z Med J 1989;102:557-8.

3. Shemer J, Modan M, Ezra D, Cabili S. Incidence of hyperkalemia in hospitalized patients. Isr J Med Sci 1983;19:659-61.

4. Muschart $X$, Boulouffe $C_{1}$ Jamart J, et al. A determination of the current causes of hyperkalaemia and whether they have changed over the past 25 years. Acta Clin Belg 2014;69:2804.

5. Fleet JL, Shariff SZ, Gandhi S, Weir MA, Jain AK, Garg AX. Validity of the International Classification of Diseases 10th revision code for hyperkalaemia in elderly patients at presentation to an emergency department and at hospital admission. BMJ Open 2012;2:e002011.

6. Ookuma T, Miyasho K, Kashitani N, et al. The clinical relevance of plasma potassium abnormalities on admission in trauma patients: a retrospective observational study. J Intensive Care 2015;3:37.

7. Khanagavi J, Gupta T, Aronow WS, et al. Hyperkalemia among hospitalized patients and association between duration of hyperkalemia and outcomes. Arch Med Sci 2014;10:251-7.

8. An JN, Lee JP, Jeon $\mathrm{HJ}$, et al. Severe hyperkalemia requiring hospitalization: predictors of mortality. Crit Care 2012;16:R225.

9. Jain N, Kotla S, Little BB, et al. Predictors of hyperkalemia and death in patients with cardiac and renal disease. Am J Cardiol 2012;109:1510-3.

10. Goyal A, Spertus JA, Gosch K, et al. Serum potassium levels and mortality in acute myocardial infarction. JAMA 2012;307: 157-64.

11. Grodzinsky A, Goyal A, Gosch K, et al. Prevalence and prognosis of hyperkalemia in patients with acute myocardial infarction. Am J Med 2016;129:858-65.

12. Marti $G$, Schwarz $C$, Leichtle $A B$, et al. Etiology and symptoms of severe hypokalemia in emergency department patients. Eur J Emerg Med 2014;21:46-51.

13. Packham DK, Rasmussen HS, Lavin PT, et al. Sodium zirconium cyclosilicate in hyperkalemia. N Engl J Med 2015;372:222-31.

14. Weir MR, Bakris GL, Bushinsky DA, et al. Patiromer in patients with kidney disease and hyperkalemia receiving RAAS inhibitors. N Engl J Med 2015;372:211-21.

15. Palmer BF. Managing hyperkalemia caused by inhibitors of the renin-angiotensin-aldosterone system. N Engl J Med 2004;351:585-92.

16. Svensson M, Gustafsson $F_{1}$ Galatius $S_{1}$ Hildebrandt PR, Atar D. How prevalent is hyperkalemia and renal dysfunction during treatment with spironolactone in patients with congestive heart failure? J Card Fail 2004;10:297-303.

17. Weinberg JM, Appel $\amalg$, Bakris $G$, et al. Risk of hyperkalemia in nondiabetic patients with chronic kidney disease receiving 
antihypertensive therapy. Arch Intern Med 2009;169:158794.

18. Gilbert EH, Lowenstein SR, Koziol-McLain J, Barta DC, Steiner J. Chart reviews in emergency medicine research: where are the methods? Ann Emerg Med 1996;27:305-8.

19. Kaji AH, Schriger D, Green S. Looking through the retrospectoscope: reducing bias in emergency medicine chart review studies. Ann Emerg Med 2014;64:292-8.

20. Guidelines 2000 for Cardiopulmonary Resuscitation and Emergency Cardiovascular Care. Part 8: advanced challenges in resuscitation: section 1: life-threatening electrolyte abnormalities. The American Heart Association in collaboration with the International Liaison Committee on Resuscitation. Circulation 2000;102:1217-22.

21. Freeman $K$, Feldman JA, Mitchell $P$, et al. Effects of presenta- tion and electrocardiogram on time to treatment of hyperkalemia. Acad Emerg Med 2008;15:239-49.

22. Acker CG, Johnson JP, Palevsky PM, Greenberg A. Hyperkalemia in hospitalized patients: causes, adequacy of treatment, and results of an attempt to improve physician compliance with published therapy guidelines. Arch Intern Med 1998;158: 917-24.

23. Hayes J, Kalantar-Zadeh K, Lu JL, Turban S, Anderson JE, Kovesdy CP. Association of hypo- and hyperkalemia with disease progression and mortality in males with chronic kidney disease: the role of race. Nephron Clin Pract 2012;120:c8-16.

24. Einhorn $L M$, Zhan $M, H s u V D$, et al. The frequency of hyperkalemia and its significance in chronic kidney disease. Arch Intern Med 2009;169:1156-62. 